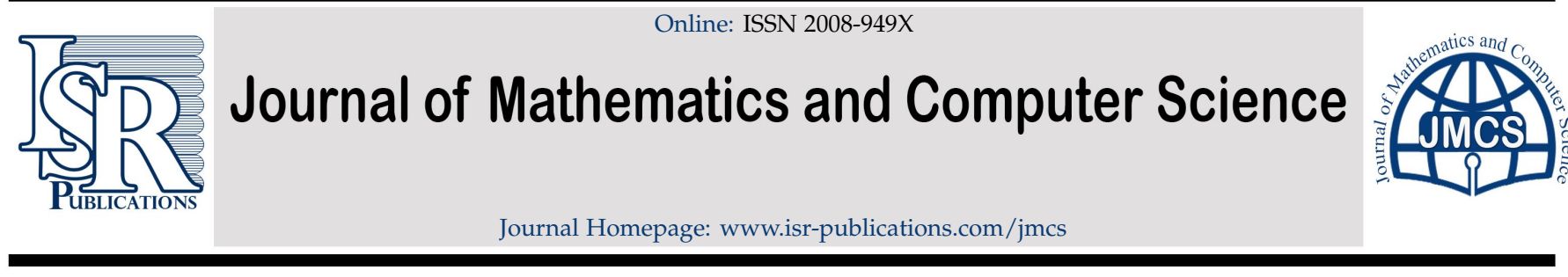

\title{
On the q-Sumudu transform with two variables and some properties
}

\author{
Artan F. Alidema a , Shkumbin V. Makollib,* \\ ${ }^{a}$ Department of Mathematics, Faculty of Mathematical and Natural Science, University of Prishtina, Mother Theresa p.n, 10000 \\ Prishtina, Kosovo. \\ ${ }^{b}$ Department of Mathematics, Faculty of Mechanical Engeenering, University of Prishtina, Agim Ramadani p.n, 1000 Pristina, Kosovo.
}

\begin{abstract}
In this paper we present some properties of double q-Sumudu transform in q-calculus by using the functions of two variables. Furthermore results on convergence, absolute convergence and convolution are discussed. At the end some examples are given to illustrate use of double q-Sumudu transform.
\end{abstract}

Keywords: Double q-Sumudu transform, convergence, convolution.

2020 MSC: 33D05, 33D60, 35A22, 42A85.

(C)2022 All rights reserved.

\section{Introduction}

The Sumudu transform was introduced by Watugala [24] and he applied it to the solution of ordinary differential equations. Asiru [7] and Belgacem [9] give the general and fundamental properties of the Sumudu transform. Watugala [25] has extended Sumudu transform to functions of two variables, and applied it to solving partial differential equations.

Tchuenche [22] applied the double Sumudu transform to an evolution equation of population dynamics. Kilicman and Gadain [19] gave the relations between double Laplace transform and double Sumudu transform and applied it to the solutions of non-homogenous wave equations. Debnath [11] presented general properties of the double Laplace transform, convolution and its properties. Convergence of double Sumudu transform was proved by Zulfiqar et al. [3].

In 1910, Jackson [17] presented a precise definition of so-called q-Jackson integral and developed qcalculus in a systematic way. It is well known that there are two types of q-Laplace transforms and they have been studied in detail by many authors $([1,16,20])$.

The theory of q-analysis has been applied in many areas of mathematics, engineering, and physics, like in ordinary fractional calculus, optimal control problems, q-transform analysis and also in finding solutions of the q-difference and q-integral equations (see $[2,8,18]$ ).

\footnotetext{
*Corresponding author

Email addresses: artan.alidema@uni-pr.edu (Artan F. Alidema), shkumbin.makolli@uni-pr.edu (Shkumbin V. Makolli)

doi: $10.22436 /$ jmcs.025.02.05
}

Received: 2021-03-19 Revised: 2021-04-12 Accepted: 2021-04-24 
Purohit and Kalla [20] evaluated the q-Laplace transforms of the q-Bessel functions, and they gave several useful cases of its application. Albayrak et al. [4, 5], have introduced q-analogue of Sumudu transform and investigated the fundamental properties of the q-Sumudu transform of certain q-polynomials. Brahim and Riah [10] introduced the q-analogue of the two dimensional Mellin transform, gave some properties and also proved the inversion formula of the q-two dimensional Mellin transform. Double q-Laplace transform was introduced by Sadjang [21] as well as by Ganie [14]. The latter gave certain results on convergence, absolute convergence, convolution and its properties.

In this paper, we examine some properties of q-double Sumudu transform in q-calculus by using functions of two variables. We have shown some results regarding the convergence, absolute convergence and introduced convolution for q-double Sumudu transform.

The Sumudu transform of function $f(x)$ is defined by Watugala in [24] as:

$$
S[f(x) ; s]=\frac{1}{s} \int_{0}^{\infty} e^{-\frac{\chi}{s}} f(x) d x, s \in\left(-\tau_{1}, \tau_{2}\right),
$$

while $f(x)$ is a function from the set of functions

$$
A=\left[f(x)\left|\exists M, \tau_{1}, \tau_{2}>0,\right| f(x) \mid<M e^{\frac{x}{\tau_{j}}}, \text { if } \quad x \in(-1)^{j} \times[0, \infty)\right] .
$$

The q-analoque of Sumudu transform by the q-Jackson integral is given by Albayrak et al. [6] as follows:

$$
F(s)=S_{q}[f(x) ; s]=\frac{1}{(1-q) s} \int_{0}^{\infty} e_{q}^{-\frac{x}{s}} f(x) d_{q} x, s \in\left(-\tau_{1}, \tau_{2}\right)
$$

Over the set of functions

$$
B=\left[f(x)\left|\exists M, \tau_{1}, \tau_{2}>0,\right| f(x) \mid<M e_{q}^{\frac{|x|}{\tau_{j}}}, \text { if } \quad x \in(-1)^{j} \times[0, \infty)\right] .
$$

Let $f(x, y)$ be the function of two variables in the positive quadrant of Oxy plane then its double Sumudu transform is given by [22] as:

$$
S[f(x, t) ;(p, s)]=\frac{1}{p s} \int_{0}^{\infty} \int_{0}^{\infty} e^{\left(-\frac{x}{p}-\frac{t}{s}\right)} f(x, t) d x d t .
$$

Double inverse Sumudu transform can be written as:

$$
S^{-1}(p . s)=\frac{1}{2 \pi i} \int_{\gamma-i \infty}^{\gamma+i \infty} e^{\frac{x}{p}} d p \frac{1}{2 \pi i} \int_{\delta-i \infty}^{\delta+i \infty} e^{\frac{t}{s}} f(p, s) d s
$$

where $f(p, s)$ is analytic functions for all $p$ and $s$ in the region defined by the inequalities $\operatorname{Re}(p) \geqslant \gamma$ and $\operatorname{Re}(s) \geqslant \delta$, while $\gamma$ and $\delta$ are real constants.

\section{Auxiliary results}

In this section we summarize the basic definitions and mathematical notations.

The $\mathrm{q}$-factorials for $\mathrm{q} \in(0,1)$ and $\mathrm{a} \in \mathrm{C}$ are defined as

$$
(a ; q)_{0}=1, \quad(a ; q)_{n}=\prod_{k=0}^{n-1}(1-a q)^{k}, n=1,2, \ldots, \quad(a ; q)_{\infty}=\lim _{n \rightarrow \infty}(a ; q)_{n}=\prod_{k=0}^{n-1}(1-a q)^{k}
$$

Also we write $[a]_{q}=\frac{1-q^{a}}{1-q},[a]_{q} !=\frac{(q ; q)_{n}}{(1-q)^{n}}, n \in N$.

The q-derivatives of a function are given by [18]

$$
\left(D_{q} f\right)(x)=\frac{(f(x)-f(q x)}{(1-q) x}, \text { if } x \neq 0, \quad\left(D_{q} f\right)(0)=f^{\prime}(0)
$$


provided that $f^{\prime}(0)$ exists. If $f$ is differentiable, then $\left(D_{q} f\right)(x)$ tends to $f^{\prime}(x)$ as $q$ tends to 1 . For $n \in N$ we have $D_{q}^{1}=D_{q},\left(D_{q}^{+}\right)^{1}=D_{q}^{+}$. The $q$-derivative of the product of two functions is defined as

$$
D_{q}(f \cdot g)(x)=g(x) D_{q} f(x)+f(q x) D_{q} g(x) .
$$

The q-integrals from 0 to $a$ and from 0 to $\infty$ known as q-Jackson integrals are defined in [17]

$$
\int_{0}^{a} f(x) d_{q}(x)=(1-q) a \sum_{n=-\infty}^{\infty} f\left(a q^{n}\right) q^{n}, \quad \int_{0}^{\infty} f(x) d_{q}(x)=(1-q) \sum_{n=-\infty}^{\infty} f\left(q^{n}\right) q^{n},
$$

provided these sums converge absolutely. The integration by parts in terms of q-calculus is given by:

$$
\int_{a}^{b} g(x) D_{q} f(x) d_{q} x=f(b) g(b)-f(a) g(a)-\int_{a}^{b} f(q x) D_{q} g(x) d_{q} x .
$$

The q-analogues of the exponential functions are defined in [15, 18],

$$
E_{q}^{z}=\sum_{n=0}^{\infty} q^{n(n-1) / 2} \frac{z^{n}}{[n]_{q} !}=(-(1-q) z ; q)_{\infty}, \quad e_{q}^{z}=\sum_{n=0}^{\infty} \frac{z^{n}}{[n]_{q} !}=\frac{1}{((1-q) z ; q)_{\infty}},|z|<\frac{1}{1-q} .
$$

The q-exponentials are analogues of classical exponential functions and satisfy the relations $D_{q} e_{q}^{z}=e_{q}^{z}$, $\mathrm{D}_{\mathrm{q}} \mathrm{E}_{\mathrm{q}}^{z}=\mathrm{E}_{\mathrm{q}}^{\mathrm{q} z}$, and $e_{\mathrm{q}}^{z} \mathrm{E}_{\mathrm{q}}^{-z}=\mathrm{E}_{\mathrm{q}}^{-z} e_{\mathrm{q}}^{z}=1$. Jacskson also defined $\mathrm{q}$-analogue of the gamma function $\Gamma(\mathrm{t})=$ $\int_{0}^{\infty} x^{\mathrm{t}-1} e^{-x} \mathrm{~d} x$, while many properties are found in $[23,26-28]$,

that satisfies

$$
\Gamma_{\mathrm{q}}(\mathrm{t})=\frac{(\mathrm{q} ; \mathrm{q})_{\infty}}{\left(\mathrm{q}^{\mathrm{t}} ; \mathrm{q}\right)_{\infty}}(1-\mathrm{q})^{1-\mathrm{t}}, \quad \mathrm{t} \neq 0,-1,-2, \ldots,
$$

$$
\Gamma_{\mathrm{q}}(\mathrm{t}+1)=[\mathrm{t}]_{\mathrm{q}} \Gamma(\mathrm{t}), \quad \Gamma_{\mathrm{q}}(1)=1,
$$

and $\lim _{\mathrm{q} \rightarrow 1^{-}} \Gamma_{\mathrm{q}}(\mathrm{t})=\Gamma(\mathrm{t}), \quad \mathbb{R}(\mathrm{t})>0$. The $\Gamma_{\mathrm{q}}$ function has the $\mathrm{q}$-integral representation as

$$
\Gamma_{\mathrm{q}}(\mathrm{s})=\int_{0}^{1 /(1-\mathrm{q})} \mathrm{t}^{\mathrm{s}-1} \mathrm{E}_{\mathrm{q}}^{-\mathrm{qt}} \mathrm{d}_{\mathrm{q}} \mathrm{t}=\int_{0}^{\infty /(1-\mathrm{q})} \mathrm{t}^{\mathrm{s}-1} \mathrm{E}_{\mathrm{q}}^{-\mathrm{qt}} \mathrm{d}_{\mathrm{q}} \mathrm{t}
$$

The q-integral representation of $\Gamma_{q}$ based on $q$-exponential function $e_{q}^{x}$ and $q$-integral representation of $q$-beta function are defined in [12] as: for all $s, t>0$, we have

$$
\Gamma_{\mathrm{q}}(\mathrm{s})=\mathrm{K}_{\mathrm{q}}(\mathrm{s}) \int_{0}^{\infty /(1-\mathrm{q})} \mathrm{t}^{\mathrm{s}-1} \mathrm{e}_{\mathrm{q}}^{-\mathrm{t}} \mathrm{d}_{\mathrm{q}} \mathrm{t}
$$

and $B_{q}(t, s)=K_{q}(t) \int_{0}^{\infty} \chi^{t-1} \frac{\left(-x q^{s+t} ; q\right)_{\infty}}{(-x ; q)_{\infty}} d_{q} x$, where in [10], $K_{q}(t)=\frac{(-q,-1 ; q)_{\infty}}{\left(-q^{t},-q^{1-t} ; q\right)_{\infty}}$. If $\frac{\log (1-q)}{\log (q)} \in Z$, we obtain

$$
\Gamma_{\mathrm{q}}(\mathrm{s})=\mathrm{K}_{\mathrm{q}}(\mathrm{s}) \int_{0}^{\infty} \mathrm{t}^{\mathrm{s}-1} e_{\mathrm{q}}^{-\mathrm{t}} \mathrm{d}_{\mathrm{q}} \mathrm{t}=\int_{0}^{\infty} \mathrm{t}^{\mathrm{s}-1} \mathrm{E}_{\mathrm{q}}^{-\mathrm{qt}} \mathrm{d}_{\mathrm{q}} \mathrm{t} .
$$

\section{Two dimensional q-Sumudu transform}

Definition 3.1. Let $q=\left(q_{1}, q_{2}\right) \in(0,1)^{2},(s, t) \in \mathbb{C}^{2}$ and let $f$ be a function of two variables $x$ and $y$ defined on $\mathbb{R}_{\mathrm{q}_{1},+} \times \mathbb{R}_{\mathrm{q}_{2},+}$. Then the $\mathrm{q}$-two dimensional Sumudu transform of $\mathrm{f}$ is defined by the double integral is given as: ([13])

$$
S_{q}(f)(s, t)=S_{q}[f(x, y)](s, t)=\frac{1}{(1-q)^{2}} \frac{1}{p s} \int_{0}^{\infty} \int_{0}^{\infty} e_{q}^{\left(-\frac{x}{p}-\frac{t}{s}\right)} f(x, t) d_{q} x d_{q} t
$$

(provided that this integral exists), where $R_{\mathrm{q},+}=\left\{q^{n}, n \in \mathbb{Z}\right\}$ and

$$
\begin{cases}e_{\mathrm{q}}^{-x}=[1-(1-q) x]^{1 / 1-q}, & \text { for } 0<x<\frac{1}{1-q}, q<1, \\ e_{q}^{-x}=[1-(1-q) x]^{-1 / 1-q}, & \text { for } x \geqslant 0, q>1\end{cases}
$$




\section{Convergence of q-double Sumudu transform}

Theorem 4.1. Let $\mathrm{f}(\mathrm{x}, \mathrm{y})$ be a function of two variables continuous in $\mathbb{R}_{\mathrm{q}_{1},+} \times \mathbb{R}_{\mathrm{q}_{2},+}$ or continuous in the positive quadrant of $x y$ plane. If the integral

$$
\frac{1}{(1-q)^{2}} \frac{1}{p s} \int_{0}^{\infty} \int_{0}^{\infty} e_{q}^{\left(-\frac{x}{p}-\frac{t}{s}\right)} f(x, t) d_{q} x d_{q} t
$$

converges at $\mathrm{s}=\mathrm{s}_{0}$ and $\mathrm{t}=\mathrm{t}_{0}$, then the integral in (4.1) converges for $\mathrm{s}<\mathrm{s}_{0}, \mathrm{p}<\mathrm{p}_{0}$.

We prove this Theorem by using following Lemma.

Lemma 4.2. If the integral

$$
\frac{1}{(1-q)} \frac{1}{s} \int_{0}^{\infty} e_{q}^{-\frac{t}{s}} f(x, t) d_{q} t
$$

converges for $\mathrm{s}=\mathrm{s}_{0}$, then the integral converge for $\mathrm{s}<\mathrm{s}_{0}$.

Proof. Let us assume that, $\alpha(x, t)=\frac{1}{(1-q)} \frac{1}{s_{0}} \int_{0}^{t} e_{q}^{\left(-\frac{u}{s_{0}}\right)} f(x, u) d_{q} u$. It is obvious that $\alpha(x, 0)=0$ and $\lim _{t \rightarrow \infty} \alpha(x, t)$ exists, because integral $\frac{1}{(1-q)} \frac{1}{s} \int_{0}^{\infty} e_{q}^{\left(-\frac{t}{s}\right)} f(x, t) d_{q} t$ converges at $s=s_{0}$.

Now let us denote

$$
\alpha_{t}(x, t)=\frac{1}{(1-q)} \frac{1}{s_{0}} e_{q}^{\left(-\frac{t}{s_{0}}\right)} f(x, t) .
$$

For $\zeta$ and $\mathrm{R}$ such that $0<\zeta<\mathrm{R}$ we will consider

$$
\begin{aligned}
\frac{1}{(1-q)} \frac{1}{s} \int_{\zeta}^{R} e_{q}^{-\frac{t}{s}} f(x, t) d_{q} t & =\frac{1}{(1-q)} \frac{1}{s} \int_{\zeta}^{R} e_{q}^{-\frac{t}{s}}(1-q) s_{0} e_{q}^{\frac{t}{s_{0}}} \alpha_{t}(x, t) d_{q} t \\
& =\frac{s_{0}}{s} \int_{\zeta}^{R} e_{q}^{-\frac{t}{s}} e_{q}^{\frac{t}{s_{0}}} \alpha_{t}(x, t) d_{q} t=\frac{s_{0}}{s} \int_{\zeta}^{R} e^{-\frac{t\left(s_{0}-s\right)}{s_{0} s}} \alpha_{t}(x, t) d_{q} t .
\end{aligned}
$$

If we use partial integration we obtain

$$
\begin{aligned}
& =\frac{s_{0}}{s}\left[\left.e_{\mathrm{q}}^{\frac{-t\left(s_{0}-s\right)}{s s_{0}}} \alpha(x, t)\right|_{\zeta} ^{R}+\frac{s_{0}-s}{s s_{0}} \int_{\zeta}^{R} e_{q}^{\frac{-t\left(s_{0}-s\right)}{s s_{0}}} \alpha(x, q t) d_{q} t\right] \\
& =\frac{s_{0}}{s}\left[e^{\frac{-R\left(s_{0}-s\right)}{s s_{0}}} \alpha(x, R)-e^{\frac{-\zeta\left(s_{0} s\right)}{s s_{0}}} \alpha(x, \zeta)+\frac{s_{0}-s}{s s_{0}} \int_{\zeta}^{R} e^{\frac{-t\left(s_{0}-s\right)}{s s_{0}}} \alpha(x, q t) d_{q} t\right] .
\end{aligned}
$$

If in the last expression we put $\zeta \rightarrow 0$, then due to $\lim _{\zeta \rightarrow 0} \alpha(x, \zeta)=\alpha(x, 0)=0$, we get

$$
\frac{1}{(1-q) s} \int_{0}^{R} e^{\frac{-t}{s}} f(x, t) d_{q} t=\frac{s_{0}}{s}\left[e^{\frac{-R\left(s_{0}-s\right)}{s s_{0}}} \alpha(x, R)+\frac{s_{0}-s}{s s_{0}} \int_{0}^{R} e^{\frac{-t\left(s_{0}-s\right)}{s s_{0}}} \alpha(x, q t) d_{q} t\right] .
$$

Now if we let $R \rightarrow \infty$, then the first term on the right tends to zero when $s<s_{0}$, subsequently we get

$$
e_{\mathrm{q}}^{\frac{-R\left(s_{0}-s\right)}{s s_{0}}} \alpha(x, R) \rightarrow 0
$$

and the left expression is equal to

$$
\frac{1}{(1-q) s} \int_{0}^{\infty} e_{q}^{\frac{-t}{s}} f(x, t) d_{q} t=\frac{s_{0}-s}{s^{2}} \int_{0}^{\infty} e_{q}^{\frac{-t\left(s_{0}-s\right)}{s s_{0}}} \alpha(x, q t) d_{q} t
$$

According to limit test for convergence of improper integrals we can prove that

$$
\frac{\left(s_{0}-s\right)}{s^{2}} \int_{0}^{\infty} e^{\frac{-t\left(s_{0}-s\right)}{s s_{0}}} \alpha(x, q t) d_{q} t
$$


converges if the following limit converges

$$
\lim _{t \rightarrow \infty} t^{p} e_{q}^{\frac{-t\left(s_{0}-s\right)}{s s_{0}}} \alpha(x, q t), \text { for, } p>1 .
$$

It is obvious that for $p=2$ and $s<s_{0}$ the above limit converges, considering the fact that $\lim _{t \rightarrow \infty} \alpha(x, q t)$ converges. Therefore the following integral

$$
\frac{1}{(1-q)} \frac{1}{s} \int_{0}^{\infty} e_{\mathrm{q}}^{-\frac{t}{s}} f(x, t) d_{q} t
$$

converges for $\mathrm{s}<\mathrm{s}_{0}$.

Lemma 4.3. If the integral

$$
\frac{1}{(1-q)} \frac{1}{s} \int_{0}^{\infty} e_{q}^{-\frac{t}{s}} f(x, t) d_{q} t
$$

converges for $\mathrm{s} \leqslant \mathrm{s}_{0}$ and the integral

$$
\frac{1}{(1-q)} \frac{1}{p} \int_{0}^{\infty} e_{q}^{-\frac{x}{p}} f(x, s) d_{q} x
$$

converges for $\mathrm{p}=\mathrm{p}_{0}$, then integral (4.2) converges for $\mathrm{p}<\mathrm{p}_{0}$.

Proof. Same as above Lemma.

Proof of the Theorem 4.1.

$$
\begin{aligned}
\frac{1}{(1-q)^{2}} \frac{1}{p s} \int_{0}^{\infty} \int_{0}^{\infty} e_{q}^{-\frac{x}{p}-\frac{t}{s}} f(x, t) d_{q} x d_{q} t & =\frac{1}{(1-q)} \frac{1}{p} \int_{0}^{\infty} e_{q}^{-\frac{x}{p}}\left\{\frac{1}{(1-q)} \frac{1}{s} \int_{0}^{\infty} e_{q}^{-\frac{t}{s}} f(x, t) d_{q} t\right\} d_{q} x \\
& =\frac{1}{(1-q)} \frac{1}{p} \int_{0}^{\infty} e_{q}^{-\frac{x}{p}} h(x, t) d_{q} x
\end{aligned}
$$

where $h(x, t)=\frac{1}{(1-q)} \frac{1}{s} \int_{0}^{\infty} e_{q}^{-\frac{t}{s}} f(x, t) d_{q} t$ and according to Lemma 4.3 it converges for $s<s_{0}$, while in accordance with Lemma 4.2 the following $\frac{1}{(1-q)} \frac{1}{p} \int_{0}^{\infty} e_{q}^{-\frac{x}{p}} h(x, t) d_{q} x$ converges for $p<p_{0}$. Therefore, the initial integral, $\frac{1}{(1-q)^{2}} \frac{1}{p s} \int_{0}^{\infty} \int_{0}^{\infty} e_{q}^{-\frac{x}{p}-\frac{t}{s}} f(x, t) d_{q} x d_{q} t$ converges for $s<s_{0}, p<p_{0}$.

Theorem 4.4 (Absolute convergence). If integral

$$
\frac{1}{(1-q)^{2}} \frac{1}{p s} \int_{0}^{\infty} \int_{0}^{\infty} e_{q}^{\left(-\frac{x}{p} \frac{-t}{s}\right)} f(x, t) d_{q} x d_{q} t
$$

converges absolute for $s=s_{0}$ and $p=p_{0}$, then it converges for $s \leqslant s_{0}$ and $p \leqslant p_{0}$.

Proof.

$$
e_{\mathrm{q}}^{\left(-\frac{x}{\mathrm{p}}-\frac{\mathrm{t}}{\mathrm{s}}\right)}|f(x, t)| \leqslant e_{\mathrm{q}}^{\left(-\frac{x}{p_{0}}-\frac{\mathrm{t}}{s_{0}}\right)}|f(x, t)| \text { for }\left(p \leqslant p_{0}<+\infty, s \leqslant s_{0}<+\infty\right),
$$

therefore

$$
\begin{aligned}
& \leqslant \frac{1}{(1-q)^{2}} \frac{1}{p s} \int_{0}^{\infty} \int_{0}^{\infty} e_{\mathrm{q}}^{\left(-\frac{x}{p}-\frac{t}{s}\right)}|f(x, t)| d_{q} t_{d_{q}} x \\
& \leqslant \frac{1}{(1-q)^{2}} \frac{1}{p s} \int_{0}^{\infty} \int_{0}^{\infty} e_{\mathrm{q}}^{\left(-\frac{x}{p_{0}}-\frac{t}{s_{0}}\right)}|f(x, t)| d_{\mathrm{q}} t d_{\mathrm{q}} x \\
& \leqslant \frac{1}{(1-q)^{2}} \frac{p_{0} s_{0}}{p s} \frac{1}{p_{0} s_{0}} \int_{0}^{\infty} \int_{0}^{\infty} e_{\mathrm{q}}^{\left(-\frac{x}{p_{0}}-\frac{t}{s_{0}}\right)}|f(x, t)| d_{\mathrm{q}} \mathrm{td}_{\mathrm{q}} x
\end{aligned}
$$

The last integral converges based on the hypothesis, which means that the initial integral converges for $p \leqslant p_{0}$ and $s \leqslant s_{0}$. 
Theorem 4.5. If $\mathrm{f}(\mathrm{x}, \mathrm{y})$ is a periodic function of periods $\mathrm{a}$ and $\mathrm{b}, \mathrm{f}(\mathrm{x}+\mathrm{a}, \mathrm{y}+\mathrm{b})=\mathrm{f}(\mathrm{x}, \mathrm{y})$, and if $\mathrm{S}_{\mathrm{q}}[\mathrm{f}(\mathrm{x}, \mathrm{y})]$ exists, then

$$
S_{q}[f(x, y)](p, s)=\frac{\left[1-e^{-\frac{a}{p}-\frac{b}{s}}\right]^{-1}}{(1-q)^{2} p s} \int_{0}^{a} \int_{0}^{b} e_{q}^{\left(-\frac{x}{p}-\frac{t}{s}\right)} f(x, t) d_{q} x d_{q} t .
$$

Proof.

$$
\begin{aligned}
S_{q}(f(x, y)) & =\frac{1}{(1-q)^{2} p s} \int_{0}^{\infty} \int_{0}^{\infty} e_{q}^{\left(-\frac{x}{p}-\frac{t}{s}\right)} f(x, t) d_{q} x d_{q} t \\
& =\frac{1}{(1-q)^{2} p s} \int_{0}^{a} \int_{0}^{b} e_{q}^{\left(-\frac{x}{p}-\frac{t}{s}\right)} f(x, t) d_{q} x d_{q} t+\frac{1}{(1-q)^{2} p s} \int_{a}^{\infty} \int_{b}^{\infty} e_{q}^{\left(-\frac{x}{p}-\frac{t}{s}\right)} f(x, t) d_{q} x d_{q} t
\end{aligned}
$$

if we put $x=u+a$ and $t=v+b$, we get

$$
=\frac{1}{(1-q)^{2} p s} \int_{0}^{a} \int_{0}^{b} e_{q}^{\left(-\frac{x}{p}-\frac{t}{s}\right)} f(x, t) d_{q} x d_{q} t+\frac{1}{(1-q)^{2} p s} \cdot e_{q}^{\frac{-a}{p}-\frac{b}{s}} \int_{0}^{\infty} \int_{0}^{\infty} e_{q}^{\left(-\frac{u}{p}-\frac{v}{s}\right)} f(u, v) d_{q} u d_{q} v .
$$

Therefore

$$
\begin{aligned}
S_{q} f(x, t) & =\frac{1}{(1-q)^{2} p s} \int_{0}^{a} \int_{0}^{b} e_{q}^{\left(-\frac{x}{p}-\frac{t}{s}\right)} f(x, t) d_{q} x d_{q} t+e_{q}^{\left(-\frac{a}{p}-\frac{b}{s}\right)} S_{q} f(x, t) \\
& =\left[1-e_{q}^{\left(-\frac{a}{p}-\frac{b}{s}\right)}\right] S_{q} f(x, t)=\frac{1}{(1-q)^{2} p s} \int_{0}^{a} \int_{0}^{b} e_{q}^{\left(-\frac{x}{p}-\frac{t}{s}\right)} f(x, t) d_{q} x d_{q} t, \\
S_{q} f(x, t) & =\frac{\left[1-e_{q}^{-\frac{a}{p}-\frac{b}{s}}\right]^{-1}}{(1-q)^{2} p s} \int_{0}^{a} \int_{0}^{b} e_{q}^{\left(-\frac{x}{p}-\frac{t}{s}\right)} f(x, t) d_{q} x d_{q} t,
\end{aligned}
$$

$\mathrm{S}_{\mathrm{q}}$ is a double Sumudu transform of a periodic function.

\section{Double q-Sumudu convolution product}

Definition 5.1. The convolution of $f(x, y)$ and $g(x, y)$ is defined as

$$
(f * * g)(x, y)=\frac{1}{(1-q)^{2} p s} \int_{0}^{x} \int_{0}^{y} f(\zeta, \mu) g(x-\zeta, y-\mu) d_{q} \zeta d_{q} \mu .
$$

Theorem 5.2 (Convolution Theorem). Let $\mathrm{f}_{1}(\mathrm{x}, \mathrm{t})$ and $\mathrm{f}_{2}(\mathrm{x}, \mathrm{t})$ be two functions having double $\mathrm{q}$-double Sumudu transform. Then q-double Sumudu transform of the double convolution is given by:

$$
\mathrm{S}_{\mathrm{q}}\left\{\mathrm{f}_{1}(\mathrm{x}, \mathrm{t}) * * \mathrm{f}_{2}(\mathrm{x}, \mathrm{t})\right\}(\mathrm{p}, \mathrm{s})=\mathrm{S}_{\mathrm{q}}\left[\mathrm{f}_{1}(\mathrm{x}, \mathrm{t})\right] \mathrm{S}_{\mathrm{q}}\left[\mathrm{f}_{2}(\mathrm{x}, \mathrm{t})\right] .
$$

Proof.

$$
\begin{aligned}
& \mathrm{S}_{\mathrm{q}}\left\{\mathrm{f}_{1}(\mathrm{x}, \mathrm{t}) * * \mathrm{f}_{2}(\mathrm{x}, \mathrm{t})\right\}(\mathrm{p}, \mathrm{s}) \\
& =\frac{1}{(1-q)^{4} p s} \int_{0}^{\infty} \int_{0}^{\infty} e_{q}^{\left(-\frac{x}{p}-\frac{t}{s}\right)}\left\{\int_{0}^{x} \int_{0}^{t} f_{1}(\zeta, \mu) f_{2}(x-\zeta, t-\mu) d_{q} \zeta d_{q} \mu\right\} d_{q} x d_{q} t \\
& =\frac{1}{(1-q)^{4} p^{2} s^{2}} \int_{0}^{\infty} \int_{0}^{\infty}\left\{\int_{0}^{x} \int_{0}^{t}\left[1+(q-1)\left(\frac{x}{p}+\frac{t}{s}\right)\right]^{-\frac{1}{q-1}} f_{1}(\zeta, \mu) f_{2}(x-\zeta, t-\mu) d_{q} \zeta d_{q} \mu\right\} d_{q} x d_{q} t \\
& =\frac{1}{(1-q)^{4} p^{2} s^{2}} \int_{0}^{\infty} \int_{0}^{\infty} f_{1}(\zeta, \mu)\left\{\int_{0}^{x} \int_{0}^{t}\left[1+(q-1)\left(\frac{x}{p}+\frac{t}{s}\right)\right]^{-\frac{1}{q-1}} f_{2}(x-\zeta, t-\mu) d_{q} \zeta d_{q} \mu\right\} d_{q} x d_{q} t .
\end{aligned}
$$

Let $u$ seplace $x-\zeta=u$ and $t-\mu=v$, and if we denote

$$
I=\int_{0}^{x} \int_{0}^{t}\left[1+(q-1)\left(\frac{x}{p}+\frac{t}{s}\right)\right]^{-\frac{1}{q-1}} f_{2}(x-\zeta, y-\mu) d_{q} \zeta d_{q} \mu
$$


in light of new variables, and if we consider upper bounds for $x$ and $t$, the integral I can also be written as following

$$
\begin{aligned}
I= & {\left[1+(q-1)\left(\frac{\zeta}{p}+\frac{\mu}{s}\right)\right]^{-\frac{1}{q-1}} \int_{-\zeta}^{\infty} \int_{-\mu}^{\infty}\left[1+(q-1)\left(\frac{u}{p}+\frac{v}{s}\right)\right]^{-\frac{1}{q-1}} } \\
& \times\left[1+(q-1)\left(\frac{\zeta}{p}+\frac{\mu}{s}\right)\right]^{\frac{1}{q-1}}\left[1+(q-1)\left(\frac{u}{p}+\frac{v}{s}\right)\right]^{\frac{1}{q-1}}\left[1+(q-1)\left(\frac{u+\zeta}{p}+\frac{v+\mu}{s}\right)\right]^{-\frac{1}{q-1}} f_{2}(u, v) d_{q} u d_{q} v .
\end{aligned}
$$

Since both functions $f_{1}(x, t)$ and $f_{2}(x, t)$ are defined in the positive quadrant of the Oxy plane, it is obvious that

$$
\begin{aligned}
I= & {\left[1+(q-1)\left(\frac{\zeta}{p}+\frac{\mu}{s}\right)\right]^{-\frac{1}{q-1}} \int_{0}^{\infty} \int_{0}^{\infty}\left[1+(q-1)\left(\frac{u}{p}+\frac{v}{s}\right)\right]^{-\frac{1}{q-1}} } \\
& \times\left[1+(q-1)\left(\frac{\zeta}{p}+\frac{\mu}{s}\right)\right]^{\frac{1}{q-1}}\left[1+(q-1)\left(\frac{u}{p}+\frac{v}{s}\right)\right]^{\frac{1}{q-1}}\left[1+(q-1)\left(\frac{u+\zeta}{p}+\frac{v+\mu}{s}\right)\right]^{-\frac{1}{q-1}} f_{2}(u, v) d_{q} u d_{q} v .
\end{aligned}
$$

If we now write

$$
\left[1+(q-1)\left(\frac{\zeta}{p}+\frac{\mu}{s}\right)\right]^{\frac{1}{q-1}}\left[1+(q-1)\left(\frac{u}{p}+\frac{v}{s}\right)\right]^{\frac{1}{q-1}}\left[1+(q-1)\left(\frac{u+\zeta}{p}+\frac{v+\mu}{s}\right)\right]^{-\frac{1}{q-1}} f_{2}(u, v)=f_{2}^{*}(u, v),
$$

from the last relation we can then express $f_{2}(u, v)$ as

$$
f_{2}(u, v)=\left[1+(q-1)\left(\frac{\zeta}{p}+\frac{\mu}{s}\right)\right]^{-\frac{1}{q-1}}\left[1+(q-1)\left(\frac{u}{p}+\frac{v}{s}\right)\right]^{-\frac{1}{q-1}}\left[1+(q-1)\left(\frac{u+\zeta}{p}+\frac{v+\mu}{s}\right)\right]^{\frac{1}{q-1}} f_{2}^{*}(u, v),
$$

and after replacing it in the initial integral we will get:

$$
\begin{aligned}
S_{\mathrm{q}}\left\{\mathrm{f}_{1}(\mathrm{x}, \mathrm{t}) * * \mathrm{f}_{2}(\mathrm{x}, \mathrm{t})\right\}= & \frac{1}{(1-\mathrm{q})^{4} \mathrm{p}^{2} \mathrm{~s}^{2}} \int_{0}^{\infty} \int_{0}^{\infty} \mathrm{f}_{1}(\zeta, \mu)\left[1+(\mathrm{q}-1)\left(\frac{\zeta}{\mathrm{p}}+\frac{\mu}{\mathrm{s}}\right)\right]^{-\frac{1}{\mathrm{q}-1}} \\
& \times\left\{\int_{0}^{\infty} \int_{0}^{\infty}\left[1+(\mathrm{q}-1)\left(\frac{\mathrm{u}}{\mathrm{p}}+\frac{v}{\mathrm{~s}}\right)\right]^{-\frac{1}{\mathrm{q}-1}} \mathrm{f}_{2}^{*}(\mathrm{u}, v) \mathrm{d}_{\mathrm{q}} u d_{\mathrm{q}} v\right\} \mathrm{d}_{\mathrm{q}} \zeta \mathrm{d}_{\mathrm{q}} \mu \\
= & \mathrm{S}_{\mathrm{q}}\left\{\mathrm{f}_{1}\{(\zeta, \mu)\}\left\{\frac{1}{(1-\mathrm{q})^{2} \mathrm{ps}} \int_{0}^{\infty} \int_{0}^{\infty}\left[1+(\mathrm{q}-1)\left(\frac{\mathrm{u}}{\mathrm{p}}+\frac{v}{\mathrm{~s}}\right)\right]^{-\frac{1}{\mathrm{q}-1}} \mathrm{f}_{2}^{*}(\mathrm{u}, v) \mathrm{d}_{\mathrm{q}} u \mathrm{~d}_{\mathrm{q}} v\right\}\right. \\
= & \mathrm{S}_{\mathrm{q}}\left\{\mathrm{f}_{1}(\zeta, \mu)\right\} \cdot \mathrm{S}_{\mathrm{q}}\left\{\mathrm{f}_{2}^{*}(\mathrm{u}, v)\right\}, \\
\mathrm{S}_{\mathrm{q}}\left\{\mathrm{f}_{1}(\mathrm{x}, \mathrm{t}) * * \mathrm{f}_{2}(\mathrm{x}, \mathrm{t})\right\}(\mathrm{p}, \mathrm{s})= & \mathrm{S}_{\mathrm{q}}\left[\mathrm{f}_{1}(\mathrm{x}, \mathrm{t})\right] \mathrm{S}_{\mathrm{q}}\left[\mathrm{f}_{2}(\mathrm{x}, \mathrm{t})\right] .
\end{aligned}
$$

\subsection{Properties of double q-Sumudu transform method}

Some properties of $q$-double Sumudu transform are given as following.

a) Scaling: For a real number $k$,

$$
\begin{aligned}
S_{q}[k f(x, y)](p, s) & =\frac{1}{(1-q)^{2}} \frac{1}{p s} \int_{0}^{\infty} \int_{0}^{\infty} k e_{q}^{\left(-\frac{x}{p}-\frac{y}{s}\right)} f(x, y) d_{q} x d_{q} y \\
& =\frac{1}{(1-q)^{2}} \frac{k}{p s} \int_{0}^{\infty} \int_{0}^{\infty} e_{q}^{\left(-\frac{x}{p}-\frac{y}{s}\right)} f(x, y) d_{q} x d_{q} y k S_{q}[f(x, y)](p, s) .
\end{aligned}
$$

\section{b) Linearity:}

$$
S_{q}\left[m f(x, y)+S_{q} m f(x, y)\right](p, s)=m S_{q}[f(x, y)]+n S_{q}[f(x, y)](p, s),
$$




$$
\begin{aligned}
S_{q}[m f(x, y)+n f(x, y)](p, s)= & \frac{1}{(1-q)^{2}} \frac{1}{p s} \int_{0}^{\infty} \int_{0}^{\infty}[m f(x, y)+n f(x, y)] e_{q}^{\left(-\frac{x}{p}-\frac{y}{s}\right)} d_{q} x d_{q} y \\
& \times \frac{1}{(1-q)^{2}} \frac{1}{p s}\left\{\int_{0}^{\infty} \int_{0}^{\infty} m f(x, y) e_{q}^{\left(-\frac{x}{p}-\frac{y}{s}\right)} d_{q} x d_{q} y\right. \\
& \left.+\int_{0}^{\infty} \int_{0}^{\infty} n f(x, y) e_{q}^{\left(-\frac{x}{p}-\frac{y}{s}\right)} d_{q} x d_{q} y\right\} \\
= & \left.m S_{q}[f(x, y)](p, s)+n S_{q}[f(x, y)](p, s)\right] .
\end{aligned}
$$

c): For $a>0, b>0$, and if we denote $\overline{\overline{G_{q}}}(p, s)=S_{q}[f(x, y)(p, s)]$, we have:

$$
\begin{aligned}
& S_{q}\left[e_{q}^{-\frac{x}{a}-\frac{y}{b}} f(x, y)\right]=\frac{a b}{(a+p)(b+s)} \overline{\overline{G_{q}}}\left(\frac{a p}{a+p}, \frac{b s}{b+s}\right), \\
& S_{q}\left[e_{q}^{-\frac{x}{a}-\frac{y}{b}} f(x, y)\right]=\frac{1}{(1-q)^{2}} \frac{1}{p s} \int_{0}^{\infty} \int_{0}^{\infty} e_{q}^{\left(-\frac{x}{p}-\frac{y}{s}\right)} e_{q}^{\left(-\frac{x}{a}-\frac{y}{b}\right)} f(x, y) d_{q} x d_{q} y \\
& =\frac{1}{(1-q)^{2}} \frac{1}{p s} \int_{0}^{\infty} \int_{0}^{\infty} e_{\mathrm{q}}^{-\frac{(\mathrm{a}+\mathrm{p}) \mathrm{x}}{\mathrm{ap}}} e_{\mathrm{q}}^{-\frac{(\mathrm{b}+\mathrm{s}) \mathrm{y}}{\mathrm{bs}}} \mathrm{f}(\mathrm{x}, \mathrm{y}) \mathrm{d}_{\mathrm{q}} x \mathrm{~d}_{\mathrm{q}} \mathrm{y} \\
& =\frac{1}{(1-q)^{2}} \frac{1}{p s} \int_{0}^{\infty} \int_{0}^{\infty} e_{q}^{-\frac{(a+p) x}{a p}-\frac{(b+s) y}{b s}} f(x, y) d_{q} x d_{q} y=\frac{a b}{(a+p)(b+s)} \overline{\overline{G_{q}}}\left(\frac{a p}{a+p}, \frac{b s}{b+s}\right) .
\end{aligned}
$$

d): $S_{q}[f(x)]=\frac{1}{(1-q)} \overline{G_{q}}(p)$, where $\overline{G_{q}}(p)=\frac{1}{(1-q)} \frac{1}{p} \int_{0}^{\infty} e_{q}^{-\frac{x}{p}} f(x) d_{q} x$,

$$
\begin{aligned}
S_{\mathrm{q}}[f(x)] & =\frac{1}{(1-q)^{2}} \frac{1}{p s} \int_{0}^{\infty} \int_{0}^{\infty} e_{\mathrm{q}}^{-\frac{x}{p}-\frac{y}{s}} f(x) d_{q} x d_{q} y \\
& =\frac{1}{(1-q)^{2}} \frac{1}{p s} \int_{0}^{\infty} e_{q}^{-\frac{y}{s}} d_{q} y \int_{0}^{\infty} e_{q}^{-\frac{x}{p}} f(x) d_{q} x \\
& =\left.\frac{1}{(1-q)^{2}} \frac{1}{p s}(-s)\left[e_{q}^{-\frac{y}{s}}\right]\right|_{0} ^{\infty} \int_{0}^{\infty} e_{q}^{-\frac{x}{p}} f(x) d_{q} x \\
& =\frac{1}{(1-q)}\left[\frac{1}{(1-q)} \frac{1}{p} \int_{0}^{\infty} e_{q}^{-\frac{x}{p}} f(x) d_{q} x\right]=\frac{1}{(1-q)} \overline{G_{q}}(p) .
\end{aligned}
$$

\subsection{Examples}

1. If $f(x, y)=1$ for $x>0, y>0$, then for $1<q<2$

$$
\begin{aligned}
& S_{\mathrm{q}}[1]=\frac{1}{(1-\mathrm{q})^{2}} \frac{1}{\mathrm{ps}} \int_{0}^{\infty} \int_{0}^{\infty} e_{\mathrm{q}}^{\left(-\frac{x}{\mathrm{p}}-\frac{\mathrm{t}}{\mathrm{s}}\right)} \mathrm{d}_{\mathrm{q}} x \mathrm{~d}_{\mathrm{q}} \mathrm{t} \\
& =\frac{1}{(1-q)^{2}} \frac{1}{p s} \int_{0}^{\infty} \int_{0}^{\infty} e_{q}^{-\frac{x}{p}} e_{q}^{-\frac{t}{s}} d_{q} x d_{q} t \\
& =\frac{1}{(1-q)^{2}} \frac{1}{p s} \int_{0}^{\infty} e_{q}^{-\frac{x}{p}}\left\{\int_{0}^{\infty}\left[1-(1-q) \frac{t}{s}\right]^{\frac{1}{1-q}} d_{q} t\right\} d_{q} x \\
& =\frac{1}{(1-q)^{2}} \frac{1}{p s} \int_{0}^{\infty} e_{q}^{-\frac{x}{p}}\left\{\left.\frac{\left[1-(1-q) \frac{t}{s}\right]^{\frac{2-q}{1-q}}}{\frac{2-q}{1-q}(1-q)} s\right|_{0} ^{\infty}\right\} d_{q} x \\
& =\frac{1}{(1-q)^{2}} \frac{1}{p} \int_{0}^{\infty} e_{q}^{-\frac{x}{p}} \frac{1}{(q-2)} d_{q} x
\end{aligned}
$$




$$
\begin{aligned}
& =\frac{1}{(1-q)^{2}} \frac{1}{p} \frac{1}{(q-2)} \int_{0}^{\infty} e_{q}^{-\frac{x}{p}} d_{q} x \\
& =\frac{1}{(1-q)^{2}} \frac{1}{p} \frac{1}{(q-2)} \int_{0}^{\infty}\left[1-(1-q) \frac{x}{p}\right]^{\frac{1}{1-q}} d_{q} x \\
& =\frac{1}{(1-q)^{2}} \frac{1}{p} \frac{1}{(q-2)}\left\{\left.\frac{\left[1-(1-q) \frac{x}{p}\right]^{\frac{2-q}{1-q}}}{\frac{2-q}{1-q}(1-q)} p\right|_{0} ^{\infty}\right\}=\frac{1}{(1-q)^{2}} \frac{1}{(q-2)^{2}} .
\end{aligned}
$$

2. If $f(x, t)=\cos _{q}\left(\frac{x}{a}+\frac{t}{b}\right)$,

$$
S_{q}\left[\cos _{q}\left(\frac{x}{a}+\frac{t}{b}\right)\right]=\frac{1}{(1-q)^{2}} \frac{1}{p s} \int_{0}^{\infty} \int_{0}^{\infty} e_{q}^{\left(-\frac{x}{p}-\frac{t}{s}\right)}\left[\frac{e_{q}^{i\left(\frac{x}{a}+\frac{t}{b}\right)}+e_{q}^{-i\left(\frac{x}{a}+\frac{t}{b}\right)}}{2}\right] d_{q} x d_{q} t .
$$

The last integral can be divided in two parts

$$
=\frac{1}{(1-\mathrm{q})^{2}} \frac{1}{p s} \int_{0}^{\infty} \int_{0}^{\infty} \frac{e_{\mathrm{q}}^{\left(-\frac{x}{\mathrm{p}}-\frac{\mathrm{t}}{\mathrm{s}}\right)} e_{\mathrm{q}}^{i\left(\frac{x}{a}+\frac{\mathrm{t}}{\mathrm{b}}\right)}}{2} \mathrm{~d}_{\mathrm{q}} \times \mathrm{d}_{\mathrm{q}} \mathrm{t}+\frac{1}{(1-\mathrm{q})^{2}} \frac{1}{p s} \int_{0}^{\infty} \int_{0}^{\infty} \frac{e_{\mathrm{q}}^{\left(-\frac{x}{p}-\frac{\mathrm{t}}{s}\right)} e_{\mathrm{q}}^{-i\left(\frac{x}{a}+\frac{\mathrm{t}}{\mathrm{b}}\right)}}{2} \mathrm{~d}_{\mathrm{q}} x \mathrm{~d}_{\mathrm{q}} \mathrm{t} .
$$

Similarly as above we get

$$
=\frac{1}{2}\left[\frac{b a}{(1-q)^{2}(b-s i)(a-p i)}+\frac{b a}{(1-q)^{2}(b+s i)(a+p i)}\right]=\frac{b a(b a-s p)}{(1-q)^{2}\left(b^{2}+s^{2}\right)\left(a^{2}+p^{2}\right)} .
$$

3. If $f(x, t)=(x t)^{n}$,

$$
\left.S_{q}\left[(x t)^{n}\right)\right]=\frac{1}{(1-q)^{2}} \frac{1}{p s} \int_{0}^{\infty} \int_{0}^{\infty} e_{q}^{\left(-\frac{x}{p}-\frac{t}{s}\right)}(x t)^{n} d_{q} x d_{q} t=\frac{1}{(1-q)^{2}} \frac{1}{p s} \int_{0}^{\infty} e_{q}^{\left(-\frac{x}{p}\right)} x^{n} d_{q} x \int_{0}^{\infty} e_{q}^{\left(-\frac{t}{s}\right)} t^{n} d_{q} t .
$$

If we substitute $\frac{x}{p}=u$, we get

$$
\begin{aligned}
& =\frac{1}{(1-q)^{2}} \frac{1}{p s} \int_{0}^{\infty} e_{q}^{-u}(p u)^{n} p d_{q} u \int_{0}^{\infty} e_{q}^{\left(-\frac{t}{s}\right)} t^{n} d_{q} t \\
& =\frac{1}{(1-q)^{2}} \frac{1}{s} p^{n} \int_{0}^{\infty} e_{q}^{-u} u^{n} d_{q} u \int_{0}^{\infty} e_{q}^{\left(-\frac{t}{s}\right)} t^{n} d_{q} t=\frac{1}{(1-q)^{2}} \frac{p^{n}}{s} \Gamma_{q}(n+1) \int_{0}^{\infty} e_{q}^{\left(-\frac{t}{s}\right)} t^{n} d_{q} t
\end{aligned}
$$

In a similar manner if we substitute $\frac{t}{s}=v$, we will get

$$
=\frac{p^{n} s^{n}}{(1-q)^{2}} \Gamma_{q}(n+1) \Gamma_{q}(n+1) \text {. }
$$

\section{Conclusion}

In this paper we have introduced some properties of double q-Sumudu transform and its convolution. Convergence and absolute convergence were also discussed, as well as the q-Sumudu transform of periodic functions. The results proved in this paper appear to be new and with certain applications to solving q-difference and q-integral equations.

\section{References}

[1] W. H. Abdi On q-Laplace Transforms, Proc. Nat. Acad. Sci. India Sect. A, 29 (1960), 389-408. 1

[2] M. H. Abu Risha, M. H. Annaby, H. E. H. Ismail, Z. S. Mansour, Linear q-difference equations, Z. Anal. Anwend., 26 (2007), 481-494. 1

[3] Z. Ahmed, M. I. Idrees, F. B. M. Belgacem, Z. Perveen, On the convergence of double Sumudu transform, J. Nonlinear Sci. Appl., 13 2019, 154-162. 1 
[4] D. Albayrak, S. D .Purohit, F. Ucar, On q-Sumudu transforms of certain q-polynomials, Filomat, 27 (2013), 411-427. 1

[5] D. Albayrak, S. D. Purohit, F. Ucar, On q-analogues of Sumudu transforms, An. Stiint. Univ. "Ovidius" Constanţa Ser. Mat., 21 (2013), 239-259. 1

[6] D. Albayrak, S. D. Purohit, F. Ucar, Certain Inversion and Representation formulas for q-Sumudu Transforms, Hacet. J. Math. Stat., 43 (2014), 699-713. 1

[7] M. A. Asiru, Further Properties of the Sumudu Transform and its Applications, Internat. J. Math. Ed. Sci. Tech., 33 (2002), 441-449. 1

[8] G. Bangerezako, Variational calculus on q-nonuniform lattices, J. Math. Anal. Appl., 306 (2005), 161-179. 1

[9] F. B. M. Belgacem, A. A. Karaballi, S. L. Kalla, Analytical investigations of the Sumudu transform and applications to integral production equations, Math. Probl. Eng., 2003 (2003), 103-118. 1

[10] K. Brahim, L. Riahi, Two dimensional Mellin transform in quantum Calculus, Acta Math. Sci. Ser. B (Engl. Ed.), 38 (2018), 546-560. 1, 2

[11] L. Debnath, The Double Laplace Transforms and Their Properties with Applications to Functional, Integral and Partial Differential Equations, Int. J. Appl. Comput. Math., 2 (2016), 223-241. 1

[12] A. De Sole, V. G. Kac, On integral representation of q-gamma and q-beta functions, Atti Accad. Naz. Lincei Cl. Sci. Fis. Mat. Natur. Rend. Lincei (9) Mat. Appl., 16 (2005), 11-29. 2

[13] J. A. Ganie, A. Ahmad, R. Jain Basic Analogue of Double Sumudu Transform and its Applicability in Population Dynamics, Asian J. Math. Stat., 11 (2018), 12-17. 3.1

[14] J. V. Ganie, R. Jain, On a system of q-Laplace transform of two variables with applications, J. Comput. Appl. Math., 366 (2020), 12 pages. 1

[15] G. Gasper, M. Rahmen, Basic Hypergeometric Series, Second ed., Cambridge University Press, Cambridge, (2004). 2

[16] W. Hahn, Beitrage Zur Theorie der Heineschen Reihen, die 24 Inte-grale der hypergeometrischen q-Diferenzengleichung, das q-Analog on der Laplace Transformation, Math. Nachr., 2 (1949), 340-379. 1

[17] D. O. Jackson, T. Fukuda, O. Dunn, E. Majors, On q-definite integrals, Quart. J. Pure Appl. Math., 41 (1910), $193-203$. 1,2

[18] V. Kac, P. Cheung, Quantum Calculus, Springer-Verlag, New York, (2002). 1, 2

[19] A. Kilicman, H. E. Gadian, On the application of Laplace and Sumudo transforms, J. Franklin Inst., 347 (2010), 848-862. 1

[20] S. D. Purohit, S. L. Kalla ,On q-Laplace transforms of the q-Bessel functions, Fract. Calc. Appl. Anal., 10 (2007), 189-196. 1

[21] P. N. Sadjang, On double q-Laplace transform and application, arXiv, 2019 (2019), 27 pages. 1

[22] J. M. Tchuenche, N. S. Mbare, An Application of the double Sumudu Transform, J. Math. Anal. Appl. (Ruse), 1 (2007), 31-39. 1

[23] M. K. Wang, Y. M. Chu, Refinements of transformation inequalities for zero-balanced hypergeometric functions, Acta Math. Sci. Ser. B (Engl. Ed.), 37 (2017), 607-622. 2

[24] G. K. Watugala, Sumudu transform: a new integral transform to solve differential equations and control engineering problems, Internat. J. Math. Ed. Sci. Tech., 24 (1993), 35-43. 1

[25] G. K. Watugula, The Sumudu transform for functions of two variables, Math. Engrg. Indust., 8 (2002), 293-302. 1

[26] Z.-H. Yang, Y.-M. Chu, Asymptotic formulas for gamma function with applications, Appl. Math. Comput., 270 (2015), 665-680. 2

[27] Z.-H. Yang, W.-M. Qian, Y.-M. Chu, W. Zhang, On rational bounds for the gamma function, J. Inequal. Appl., 2017 (2017), 17 pages.

[28] Z.-H. Yang, W. Zhang, Y.-M. Chu, Sharp Gautschi inequality for parameter $0<p<1$ with applications, Math. Inequal. Appl., 20 (2017), 1107-1120. 2

[29] T.-H. Zhao, Y.-M. Chu, H. Wang, Logarithmically complete monotonicity properties relating to the gamma function, Abstr. Appl. Anal., 2011 (2011), 13 pages. 